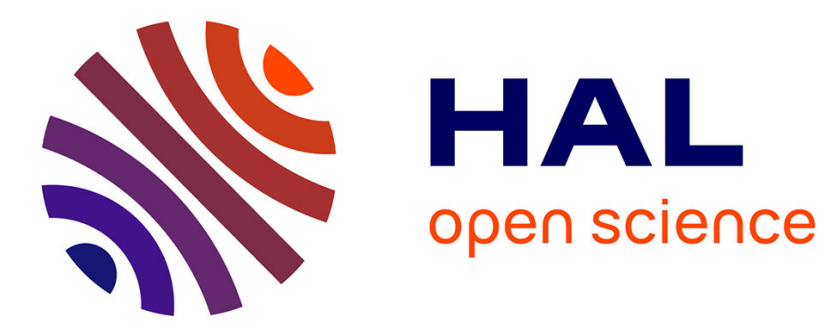

\title{
Le travail métropolitain : un outil géographique pour révéler l'usage sélectif de la grande vitesse \\ Olivier Klein
}

\section{To cite this version:}

Olivier Klein. Le travail métropolitain : un outil géographique pour révéler l'usage sélectif de la grande vitesse. Espace Géographique, 2003, 32 (2), pp. 113-127. halshs-00069050

\section{HAL Id: halshs-00069050 \\ https://shs.hal.science/halshs-00069050}

Submitted on 26 Jul 2012

HAL is a multi-disciplinary open access archive for the deposit and dissemination of scientific research documents, whether they are published or not. The documents may come from teaching and research institutions in France or abroad, or from public or private research centers.
L'archive ouverte pluridisciplinaire HAL, est destinée au dépôt et à la diffusion de documents scientifiques de niveau recherche, publiés ou non, émanant des établissements d'enseignement et de recherche français ou étrangers, des laboratoires publics ou privés. 


\section{Le travail métropolitain : un outil géographique pour révéler l'usage sélectif de la grande vitesse.}

Proposition d'article à l'Espace Géographique

Olivier KLEIN, enseignant-chercheur

Ecole Nationale des Travaux Publics de l'Etat Laboratoire d'Economie des Transports

rue Maurice Audin - 69518 Vaulx-en-Velin cedex

tel : (33) 0472047718 fax : (33) 0472047092

mél : olivier.klein@entpe.fr 


\section{Le travail métropolitain : un outil géographique pour révéler l'usage sélectif de la grande vitesse.}

\section{Mots-Clés :}

Métropolisation, travail métropolitain, train à grande vitesse, déplacements d'affaires

\section{Résumé :}

Ce papier propose une relecture de résultats d'enquêtes de mobilité réalisées autour de deux lignes ferroviaires à grande vitesse à l'aide de la notion de travail métropolitain développée par l'équipe "Géographie du système productif" du Laboratoire STRATES. II montre tout d'abord comment l'utilisation de cette segmentation de la population active, plutôt que la PCS, permet de mettre en évidence un aspect dynamique des phénomènes de différenciation sociale attachés à la métropolisation et la globalisation. La seconde partie du travail consiste à spatialiser ces résultats. On voit alors apparaître, à travers l'analyse des trafics d'affaires, une structure urbaine qui traduit à la fois le fonctionnement en réseau hiérarchisé des activités métropolitaines et la domination de l'espace métropolitain sur l'espace banal. La notion de travail métropolitain permet donc de retrouver, à partir de données qui n'ont pas été spécialement apprêtées, une image très proche de celle proposée par les principaux analystes du phénomène métropolitain. Elle se révèle un outil à la fois très opérationnel et puissant d'analyse spatiale.

\section{High speed and social hierarchy: metropolitan work as spatial analysis tool}

Key-words :

Métropolization, métropolitan work, high speed train, business traffics

\section{Summary :}

The concept of metropolitan work developed by the "Geography of the productive system" of the Laboratory STRATES is used in this paper for a second reading of mobility surveys results carried out around two high speed railway lines. Firstly, this segmentation of the working population, rather than the GCV, highlights a dynamic of the phenomena of social differentiation linked to the metropolisation and the globalisation. The second part of work consists in spatializing these results. The business traffics analyse shows an inter-urban structure which represents both a hierarchical network of the metropolitan activities and the domination of metropolitan space on banal space. Starting from data which aren't especially prepared, the concept of metropolitan work allows to find an figure very close to that proposed by the principal analysts of the metropolitan phenomenon. It appears a tool very operational and powerful of spatial analysis. 


\section{Introduction}

Dans la littérature, globalisation et métropolisation sont souvent associées à une redéfinition des hiérarchies sociales, voire un renforcement des inégalités (SASSEN, 1991 ; VELTZ, 1996). Dans le même temps, les segmentations unidimensionnelles traditionnellement utilisées pour décrire l'organisation socio-économique (la PCS ou la répartition par secteur d'activité) ne paraissent pas être en mesure de rendre compte directement et complètement des mutations contemporaines. Plusieurs tentatives de croisement de ces deux dimensions ont été proposées. On s'appuiera ici sur une distinction entre travail métropolitain et travail non métropolitain construite il y a quelques années par l'équipe "Géographie du système productif" du Laboratoire STRATES (1989) à partir des spécificités de l'économie francilienne.

L'application de cet appareillage statistique aux données d'observation de la mobilité à grande vitesse qui ont pu être recueillies dans diverses enquêtes permet de donner une nouvelle lecture des phénomènes de différenciation sociale liés à l'usage du TGV. En premier lieu, la notion de travail métropolitain met en lumière des processus ségrégatifs que le découpage par PCS ne révélait que partiellement. En outre, l'interprétation des résultats à travers cette notion permet de mieux comprendre les évolutions de mobilité constatées en les reliant non plus seulement à des paramètres relatifs à l'offre de transport - les tarifs par exemple - mais aussi à des évolutions socio-économiques exogènes au système de transport.

Après cette première lecture diachronique des résultats d'enquête, cet article explore ensuite la possibilité d'une désagrégation géographique des données. On vérifie alors que les flux de voyageurs renvoient une image plutôt fidèle de la trame urbaine telle qu'elle peut être décrite dans le paradigme métropolitain. Leur analyse fait apparaître selon des modalités différentes les phénomènes de domination des espaces économiques non métropolitains par les fonctions métropolitaines d'une part et les phénomènes de hiérarchisation du réseau de métropoles d'autre part.

Ce travail appelle deux conclusions différentes. D'abord il alimente la réflexion selon laquelle les évolutions de la mobilité que l'on constate à la faveur de la mise en service des grands équipements de transport ne peuvent pas être interprétées sans faire référence de manière prépondérante à des évolutions socio-économiques exogènes au système de transport. En ce sens, il participe à construire une lecture alternative à celles, trop déterministes, en termes « d'effet » ou « d'impact » de l'offre de transport ou des politiques publiques afférentes. En second lieu, il souligne le double intérêt problématique et méthodologique de la notion de travail métropolitain pour la compréhension des phénomènes spatialisés de différenciation: problématique, car la segmentation qui en découle n'est pas posée a priori mais possède un sens au regard des mutations du monde contemporain; méthodologique, car elle paraît suffisamment robuste pour pouvoir être utilisée sur des données qui n'ont pas été recueillies dans cette optique.

\section{Globalisation et métropolisation: renouvellement et accentuation des hiérarchies sociales}

À travers les mutations socio-économiques qu'elles traduisent, globalisation et métropolisation sont, pour de très nombreux auteurs, au cœur d'un mouvement profond de renouvellement des dynamiques de hiérarchisation sociale. Les lectures très diverses de ce phénomène permettent d'en envisager les principaux aspects.

Paul KRUGMAN analyse l'évolution des inégalités comme un processus spécifique d'élargissement la sphère d'influence des individus via les technologies de 
l'information et de la communication (1996, p. 191) ; là où, par exemple, des acteurs de théâtre ne se produisaient que devant quelques centaines de spectateurs, certains d'entre eux font aujourd'hui des shows télévisés suivis par des millions de personnes; ce processus, effet quantitatif du progrès technologique, vient creuser les hiérarchies de revenu à l'intérieur d'une même profession, au profit des « manipulateurs de symboles » (REICH, 1991).

Dans cette lignée, Daniel COHEN corrobore ce constat « d'un éclatement des inégalités au sein de chaque groupe socioculturel » (1997, p. 78). Cependant, c'est un effet qualitatif qu'il avance pour expliciter comment le phénomène est déterminé par la « troisième révolution industrielle ». Partant de l'image d'un simple joint torique qui, défaillant, a provoqué la perte de la navette spatiale Challenger et de son équipage, il exprime l'idée selon laquelle la moindre incohérence de qualité provoque aujourd'hui des coûts faramineux pour les organisations productives. Les organisations productives, de plus en plus souvent confrontées à ce type de risque, deviennent alors fortement ségrégatives: «les meilleurs vont ensemble, les médiocres aussi », avec des inégalités importantes à compétences pourtant a priori équivalentes (1997, p. 76 et suiv.). Ce regard tourné vers l'intérieur de la firme s'appuie sur une analyse classique de la sortie du fordisme (COHEN, 1994) et de la pression à l'innovation qu'induisent l'accentuation de la concurrence et la tendance au pilotage du marché « par la demande » pour comprendre l'importance nouvelle de ce qui apparaît comme une « prime à la qualité ».

C'est plutôt Saskia SASSEN (1991) qui, reprenant ce niveau d'analyse macroéconomique, établit un lien entre ces évolutions et le phénomène spécifique de métropolisation. En se globalisant et se complexifiant, le jeu économique porte en lui de nouvelles exigences de coordination auxquelles répond le phénomène métropolitain. La dispersion géographique croissante des activités et la mobilité accrue du capital - alors que la propriété demeure concentrée - ainsi que les instabilités temporelles de toutes natures « impliquent la mise sur pied d'un système de fourniture d'inputs tels que planification, gestion au plus haut niveau et services d'affaires spécialisés » (p. 69) dont les services financiers font figure d'archétype. L'ensemble de ces activités est en outre à l'origine d'un flux tout à fait essentiel d'innovations, qui alimente de manière notable leur croissance.

Ces outils de la globalisation économique se mettent en place au sein des grandes métropoles à travers l'activité de personnels de haute compétence et bénéficiant de revenus élevés, les yuppies de la finance dans l'imaginaire collectif. Ceux-ci bénéficie à la fois de la ségrégation par la qualité décrite par Daniel COHEN et de la ségrégation par la quantité reprise par Paul KRUGMAN. C'est à travers son exigence intense de coordination centralisée dans un contexte de forte complexité des organisations économiques que la globalisation est à la base d'une nouvelle dynamique - inégalitaire - de métropolisation autour du développement des activités de services sophistiqués aux entreprises.

\section{Des outils de mesure adaptés au contexte métropolitain}

La mesure des évolutions sociales résultant des mouvements de globalisation et de métropolisation se heurte pour partie à l'inefficience des outils statistiques usuels, mal adaptés à ce nouveau contexte. L'hétérogénéité du secteur tertiaire (BRAIBANT, 1982) a ainsi amené Jean GOTTMANN à proposer, dès les années 60, d'isoler en son sein - avec quelques « repêchages » depuis des secteurs d'activités industrielles à fort contenu technologique - un secteur «quaternaire » dédié aux activités « transactionnelles » (GOTTMANN, 1961 et 1970 ; COREY, 1982). II s'agit des activités 
liées au contrôle, à la prise de décision et aux transactions. Fortes utilisatrices d'informations et de savoir, ces activités se révèlent correspondre assez exactement aux services de haut niveau et aux activités directionnelles ou technologiques qui sont au cœur de toutes les analyses des processus de globalisation et de métropolisation. La difficulté est alors de trouver la base statistique sur laquelle fonder une telle distinction, sachant que ni les firmes, ni les catégories d'emplois ne sont homogènes de ce point de vue.

Les tentatives initiées pour dépasser les limites des catégories statistiques habituelles opèrent souvent un croisement entre secteur d'activité et emploi individuel afin de contourner les frontières sectorielles et de renforcer l'homogénéité interne de chaque groupe en isolant les fonctions les plus qualifiées. Mais les nouvelles catégories ainsi générées ne sont plus définies a priori : elles résultent d'une analyse précise de situations observées à partir d'une problématique spécifique. Ce faisant, ces caractéristiques produisent des jeux de données susceptibles de révéler à la fois des phénomènes de spécialisation fonctionnelle et des processus de différentiation sociale. Elles apparaissent alors en mesure d'alimenter les analyses qui abordent, à travers les questions de métropolisation, celles ayant trait à l'évolution des hiérarchies sociales.

Les traitements de données d'observation menées en lien avec l'analyse des phénomènes de métropolisation sont nombreux. On peut mentionner les travaux de Philippe JULIEN (1995) concernant le repérage des «fonctions stratégiques » en termes de dynamiques locales de l'emploi. L'analyse des processus de « gentrification » des quartiers centraux des plus grandes agglomérations est un autre domaine d'application (BEAUREGARD, 1990 ; MCDOWELL, 1997 ; PETSIMERIS et BALL, 2000). Le présent travail s'appuie quant à lui sur une recherche menée il y a déjà une dizaine d'année par une équipe de STRATES à propos des liens entre métropolisation et internationalisation de l'économie.

La méthodologie développée à cette occasion a ensuite été systématisée par le développement d'une «grille structurale-fonctionnelle » (BECKOUCHE et DAMETTE, 1993), puis utilisée pour qualifier les différentes agglomérations du système urbain français (DAMETTE, 1994). La démarche s'est appuyée sur la position particulière de l'agglomération parisienne, considérée comme l'archétype de la métropole. Les spécificités en termes d'activité et d'emploi de cet archétype ont alors été repérées et considérées comme caractéristiques de l'activité métropolitaine. Elles ont servi de base à la construction des notions de travail métropolitain et travail non métropolitain présentées dans l'encadré 2.

\section{Les enquêtes-TGV : une image des flux métropolitains}

Outre les nouveaux facteurs de différenciation sociale dont elle est porteuse, l'organisation métropolitaine se distingue aussi par une inscription particulière dans l'espace géographique. Dès l'origine, Jean GotTMANN avait constaté que les activités transactionnelles étaient très interdépendantes (GoTTMANN 1970). Depuis, un large consensus est apparu autour de l'idée d'une organisation urbaine relativement moins liée aux territoires qui lui sont immédiatement adjacents. En revanche, les moyens de communication avec les autres métropoles sont toujours considérés comme primordiaux. En fait, les métropoles sont généralement appréhendées comme les nœuds d'un réseau hiérarchisé (CASTELLS, 1996, chapitre VI, pp. 425-480 ; FANSTEIN et HARLE, 1996 ; CATTAN et alii, 1994) parcouru par d'intenses flux d'information. Complétant ce dispositif, on peut proposer l'image d'un réseau métropolitain 
dominant un «espace banal » sur lequel sont déployées les activités non métropolitaines (KLEIN, 2001, chapitre neuf).

\section{Encadré 1 : Les enquêtes TGV}

Par deux fois à l'occasion de la mise en service d'une ligne ferroviaire à grande vitesse, le Laboratoire d'Economie des Transports a pu mettre en œuvre un dispositif cohérent d'observation des évolutions de la mobilité à travers la réalisation de deux vagues d'enquête, l'une juste avant et l'autre quelques années après l'apparition du TGV. Les résultats de ces deux vagues, obtenus à chaque fois sur des périmètres géographiques et temporels semblables, peuvent alors donner lieu à comparaison pour mesurer des évolutions en volume, et de manière plus pertinente en structure, des flux de voyageurs.

Les enquêtes sud-est, réalisée essentiellement sur l'axe Paris-Lyon, ont donc été menées en septembre 1980 et en septembre 1985 (mise en service partielle du TGV en 1981 puis complète en 1983) auprès de voyageurs se déplaçant pour un motif professionnel en train ou en avion. On trouvera une description sommaire du dispositif d'observation et les principaux résultats de ces enquêtes dans (BONNAFOus, 1987) et dans (PLASSARD, 1987).

Les enquêtes Atlantique ont concerné une zone plus large incluant la totalité des relations entre Paris et l'ouest/sud-ouest de la France. Elles ont été réalisées en septembre 1989 et en septembre 1993 (mise en service partielle du TGV-A en 1989 puis complète en 1990) auprès de la totalité des voyageurs se déplaçant en train, en avion ou en voiture sur autoroute pour un motif quelconque. Pour la méthode et les résultats, on se reportera à (KLEIN et CLAISSE, 1997).

Toutes ces enquêtes relevaient les caractéristiques du déplacement observés, le motif de manière très détaillée, quelques informations sur l'entreprise d'appartenance du voyageur à motif professionnel et les principales données socio-démographiques le concernant.

Les résultats présentés ici ne concernent, pour les enquêtes TGV-A, que les déplacements à motif professionnel. La Carte 1 détaille les départements dont les trafics avec l'île-de-France ont été observés.

Dans ce cadre, la caractéristique générale des moyens de communication qui relient les nœuds de ce réseau est leur tendance à effacer la distance. Cette tendance traduit des impératifs organisationnels forts visant à libérer les acteurs économiques d'un maximum de contraintes géographiques. Concernant les réseaux de transport d'information, elle se traduit aussi de manière évidente par des impératifs techniques touchant à la vitesse et au débit. À côté des systèmes de télécommunication, les moyens de transport rapides - l'avion en premier lieu et le TGV sur de plus courtes distances - semblent donc devoir jouer un rôle particulier dans le fonctionnement du réseau métropolitain.

L'analyse des flux de déplacements transitant par les moyens de transports rapides au moyen d'une segmentation isolant les activités métropolitaines des activités banales donne une image au moins partielle du fonctionnement du réseau métropolitain. Elle présente à ce titre un double intérêt. D'une part, elle permet de vérifier la pertinence de cette représentation en termes de réseau et accessoirement, du rôle qui serait dévolu à tel ou tel mode de transport. D'autre part, à travers la distribution des flux, elle permet d'observer la répartition spatiale de l'activité métropolitaine, son éventuelle hiérarchisation et le phénomène de domination de l'espace banal. C'est avec cet objectif que la segmentation proposée par l'équipe STRATES a été utilisée ici pour réinterpréter des résultats d'enquêtes de mobilité obtenues autour de deux mises en service de lignes ferroviaires à grande vitesse. 


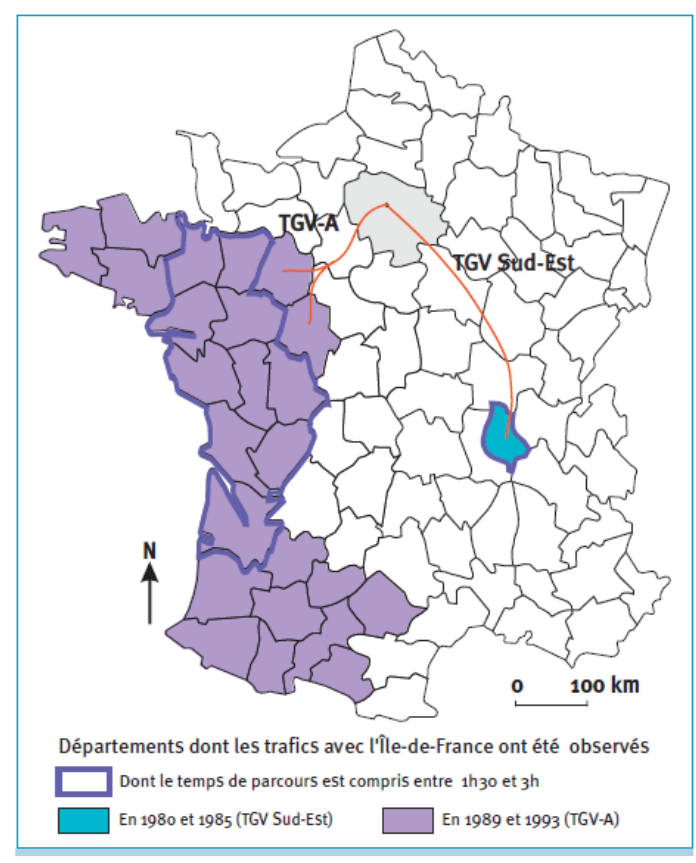

Figure 1 : les aires des enquêtes TGV

\section{Un usage socialement sélectif}

Lors de la mise en service du TGV sud-est, la SNCF avait abondamment communiqué sur le thème de la « démocratisation » de la vitesse induite par un tarif presque inchangé par rapport à celui pratiqué auparavant sur la ligne classique. Cette modération tarifaire a été rendue possible pour partie par les gains de productivité qui découlaient des choix techniques contenus dans le concept initial, pour partie encore par les économies avérées que représente l'adoption d'un tracé plus « tendu » entre Paris et Lyon et donc de presque 15\% plus court (438 km contre 512). On peut aussi parier que la nécessité d'imposer dans le public un moyen de transport nouveau a conduit la société nationale à adopter une grande prudence commerciale. La stabilité des prix, après plus de dix ans de combat pour convaincre le corps social des bienfaits de la réalisation du projet (FoURNIAU, 1988, MEUNIER, 2002), apparaît comme un ultime argument mis en œuvre par ses promoteurs propre à désarmer les oppositions. Concernant les déplacements d'affaires, la comparaison des deux enquêtes de mobilité déjà évoquées permet d'observer cette relative « démocratisation » et son évolution.

Tableau 1 : Structure et évolution de la composition socio-professionnelle du trafic d'affaires tout mode* sur les axes desservis par le TGV. Résultats d'enquêtes avant-après

\begin{tabular}{lcccccc} 
& & PCS+ & PCS- & autres & & PIB \\
\hline enquêtes & $\mathbf{1 9 8 0}$ & $80 \%$ & $13 \%$ & $7 \%$ & & $+1,6 \%$ \\
TGV sud-est & $\mathbf{1 9 8 5}$ & $75 \%$ & $18 \%$ & $7 \%$ & & $+1,9 \%$ \\
& $\mathbf{8 5 / 8 0}$ & $+26 \%$ & $+92 \%$ & $+33 \%$ & & \\
\hline enquêtes & $\mathbf{1 9 8 9}$ & $76 \%$ & $16 \%$ & $8 \%$ & & $+4 \%$ \\
TGV-A & $\mathbf{1 9 9 3}$ & $81 \%$ & $16 \%$ & $3 \%$ & & $-1,2 \%$ \\
\cline { 1 - 3 } & $\mathbf{9 3 / 8 9}$ & $+15 \%$ & $+3 \%$ & $-37 \%$ & \\
\cline { 1 - 2 } & *: Air+Fer pour le TGV S-E, Air+Fer+Route pour le TGV-A &
\end{tabular}


Le tableau 1 est construit selon la profession des individus en distinguant les positions sociales les plus élevées (PCS+: Propriétaires non salariés d'une entreprise, Professions libérales, Cadres supérieurs, Cadres, Professeurs) et les moins élevées (PCS- : Ouvriers, Employés, Techniciens, Maîtrise, Instituteurs). La catégorie «autres » rassemble principalement les commerçants et artisans. II indique la part relative de chaque catégorie dans le trafic total et l'évolution en volume de chaque segment de trafic. Pour le TGV-A, ces résultats ne concernent que la zone, desservie par TGV en 1993 en des temps de parcours compris entre 1 h30 et $3 \mathrm{~h}$, jugée la plus comparable à la situation lyonnaise (voir Carte 1). Le tableau 1 précise aussi le taux annuel d'évolution du PIB constaté en France pour l'année de chaque enquête.

Les chiffres présentés confirment en premier lieu le poids déterminant des catégories socioprofessionnelles élevées dans la mobilité d'affaires ${ }^{1}$. En second lieu, concernant le seul axe sud-est, les résultats indiquent un dynamisme indéniable de la mobilité des catégories sociales les moins favorisées qui paraît justifier l'évocation d'une «démocratisation » de la vitesse. En l'absence de chiffres comparables concernant le trafic autoroutier, il convient cependant d'être mesuré quant à la portée de ces observations. On peut toutefois souligner qu'à l'occasion de la mise en service du TGV sud-est, la régularité historiquement vérifiée (KLEIN, 2001, chapitre deux) d'un accaparement par les classes sociales les plus privilégiées des moyens de transport les plus performants ne s'est pas trouvée confirmée.

Les résultats obtenus sur la façade Atlantique montrent au contraire une différenciation sociale accentuée à la suite de l'introduction de la grande vitesse. Cette tendance, manifeste concernant les déplacement d'affaires, est confirmée sur d'autres segments de trafic (KLEIN et CLAISSE, 1997). Elle est en partie expliquée par la très nette dégradation de la conjoncture économique générale d'une vague d'enquête à l'autre. Mais, pour interpréter ces écarts, il convient aussi de souligner que, tant pour le TGV sud-est que pour l'Atlantique, la période sur laquelle ont pu être observées les modifications de l'offre de transport est courte relativement à la régularité historique évoquée ci-dessus. Elle ne permet pas de s'abstraire des éléments conjoncturels tenant à l'évolution des choix de politique tarifaire de la SNCF (tarification volontairement attractive les premières années du TGV sud-est, et délibérément restrictive pour les débuts du TGV-A). Or, les éléments tarifaires restent d'autant plus déterminants que les catégories de voyageurs dont on compare la mobilité se distinguent en premier lieu par leur revenu (apprécié ici à travers la PCS).

Afin de vérifier si l'usage de la grande vitesse ferroviaire traduit le renforcement, et surtout le renouvellement, des inégalités que les analystes rattachent à la globalisation, on va donc tenter d'emprunter une autre voie en segmentant la population active autour de la notion de travail métropolitain, selon des critères qui ne sont pas directement liés au revenu, mais plutôt au contenu informationnel des emplois. La définition de la notion, le principe du découpage et son application aux données rassemblées lors des enquêtes-TGV sont exposés dans l'encadré 2.

\footnotetext{
${ }^{1}$ Il convient cependant de souligner que les données recueillies sur trois modes de transport dans la zone Atlantique ne sont pas directement comparables en structure à celles obtenues sur deux modes entre Paris et le sud-est. Le mode routier, en particulier, apparaît plutôt plus diversifié socialement et moins « métropolitain ».
} 


\section{Tableau 2 : Structure et évolution du trafic d'affaires tout mode* selon le caractère métropolitain de l'activité des voyageurs}

\begin{tabular}{lccc} 
& & W métrop. & W non-métrop. \\
\hline enquêtes & $\mathbf{1 9 8 0}$ & $63 \%$ & $37 \%$ \\
TGV sud-est & $\mathbf{1 9 8 5}$ & $63 \%$ & $37 \%$ \\
& $\mathbf{8 5 / 8 0}$ & $+33 \%$ & $+34 \%$ \\
\hline enquêtes & $\mathbf{1 9 8 9}$ & $57 \%$ & $43 \%$ \\
TGV-A & $\mathbf{1 9 9 3}$ & $66 \%$ & $34 \%$ \\
\multicolumn{1}{c}{ *: Air+Fer pour le TGV sud-est, Air+Fer+Route pour le TGV-A }
\end{tabular}

Le travail métropolitain étant isolé au sein de la population de voyageurs observée, on mesure en premier lieu dans le tableau 2 sa prédominance au sein des flux de circulation. A contrario, on note aussi, en rappelant les réserves de comparabilité exposées en note ${ }^{1}$, que le travail non-métropolitain représente quant à lui un tiers du trafic d'affaires et n'est donc pas marginal.

L'objectif du tableau 2 est de vérifier si la grande vitesse ferroviaire participe de manière spécifique au développement des activités proprement métropolitaines en induisant une croissance particulière de la mobilité qu'elles génèrent. Entre l'enquête sud-est menée en 1980-1985 et l'enquête Atlantique de 1989-1993, les résultats sont plutôt contrastés de ce point de vue. Tout se passe comme si, le phénomène de métropolisation, encore fragile et contrarié au début des années 80 , s'était affirmé depuis.

Concernant le TGV-A tout d'abord, les résultats présentés ci-dessus semblent confirmer l'hypothèse d'une différenciation sociale spécifique, repérable autour de l'usage des moyens de transport les plus rapides par les ressortissants de l'activité métropolitaine. Entre le taux de croissance 89/93 des deux groupes distingués, le contraste est effectivement important. L'écart est plus important que celui mis en évidence avec une segmentation plus traditionnelle selon la PCS. La différence d'évolution constatée sur le trafic tout mode est encore renforcée si l'on examine le seul trafic ferroviaire. Les chiffres détaillés par mode tendraient même à révéler une capacité du TGV à induire du trafic sur le seul segment du travail métropolitain. À travers cette image, l'usage de la grande vitesse ferroviaire apparaît bien traduire le renouvellement des dynamiques de hiérarchisation sociale consécutif au phénomène de globalisation.

En regard de la netteté des évolutions affectant le TGV-A, le critère métropolitain n'apparaît absolument pas discriminant sur les résultats concernant le TGV sud-est puisque la croissance $80 / 85$ de chacun des deux segments est identique. Pour mieux comprendre, il est néanmoins nécessaire de pousser davantage l'analyse. On peut tout d'abord comparer ces résultats avec ceux du tableau 1, issus d'une segmentation sur la PCS. On remarque ainsi que l'écart entre les niveaux de mobilité du travail métropolitain et du travail non-métropolitain perdure (puisque les taux de croissance sont identiques) alors que les différences plus traditionnelles constatées entre classes de PCS, observées sur le même échantillon, semblaient largement s'estomper. En ce sens, ces résultats sont cohérents avec ceux du TGV-A qui indiquent une accentuation des différences lorsque l'on passe du critère de la PCS à celui de la « métropolité ». 
La démarche de définition du travail métropolitain est exposée par Jeannine COHEN et Félix DAMETTE (Laboratoire StRATES, 1989, pp. 3-23 puis 27-32). Elle consiste en un double découpage fonctionnel et sectoriel qui aboutit à distinguer d'une part les «fonctions abstraites » (administration-gestion, commerce, conception) des «fonctions concrètes » (fabrication, manutention-transport, services) et d'autre part la «sphère productive » de la « reproduction sociale ». Le découpage est encore affiné en isolant au sein de la sphère productive le « péri-productif » tout d'abord, constitué des « services directement nécessaires à l'activité économique », et les activités présentant un taux d'encadrement important ensuite, révélant l'importance de la part des opérations de conception en leur sein. Le poids de l'agglomération parisienne, prise comme « référent de la métropole » est enfin apprécié dans ces différents segments. II permet de définir « le travail métropolitain [comme étant] constitué par les fonctions abstraites du péri-productif et des industries conceptionnelles ».

Ce découpage est appuyé sur un appareil statistique conséquent qui réunit des données de recensement concernant les individus et des données d'entreprise provenant en particulier des enquêtes "structure des emplois » de l'INSEE. L'application de ce découpage aux données rassemblées à travers les enquêtes de mobilité réalisées autour des TGV sud-est et Atlantique se révèle délicat pour deux raisons : il est clair, d'une part, qu'une enquête embarquée dont l'unité d'observation est un déplacement ne permet de recueillir que des informations très générales sur l'emploi et a fortiori sur l'entreprise de la personne interrogée ; d'autre part, les enquêtes TGV n'ont en aucune manière été conçues pour repérer de façon précise le travail métropolitain dont le concept n'était pas apparu au moment de la rédaction des questionnaires. Dans ces conditions, il n'est pas possible de respecter toute la rigueur de la définition du travail métropolitain avancée par Félix DAMETTE pour mesurer son poids et son évolution dans la mobilité d'affaire.

Toutefois, le matériau disponible, qui contient l'indication du secteur d'activité du voyageur observé, permet cependant d'approcher cette notion de manière relativement satisfaisante, en croisant simplement cette variable avec la PCS. Selon les indications de l'équipe du laboratoire STRATES, on peut en particulier distinguer sans difficulté le secteur "péri-productif et les industries conceptionnelles » $\left(^{*}\right)$. Suivant l'atlas La France en villes (DAMETTE, 1994), il semble ensuite préférable d'agréger à cet ensemble quelques activités de «reproduction sociale élargie » qui apparaissent typiquement métropolitaines comme la recherche, les services culturels et d'administration centrale. Afin d'approcher les «fonctions abstraites » de ces activités, on exclut simplement du travail métropolitain les artisans-commerçants, les employés, les ouvriers, les policiers-militaires, les instituteurs, les membres du clergé, les étudiants, les retraités et les personnes sans profession. Cet élargissement de la notion de travail métropolitain rapproche la définition de celui-ci de la notion « d'activités transactionnelles » que Jean GOTTMANN propose dès 1961 d'isoler dans un « secteur quaternaire » (GOTTMANN, 1961, pp. 576-580 ; GOTTMANN, 1970, pp. 324-325).

Dans ces conditions, on segmente la population des voyageurs observés lors des deux enquêtes TGV en deux groupes rassemblant respectivement environ deux tiers de l'effectif sur le travail métropolitain et un tiers sur le travail non-métropolitain (alors que la part du travail métropolitain dans la population active peut être estimée à environ $10 \%$ sur la France entière ; on mesure ainsi combien les trafics ne sont pas représentatifs de la population). Le critère du revenu reste présent, mais atténué, dans cette segmentation puisque les PCS les plus élevées se retrouvent majoritairement au sein du travail métropolitain mais qu'un tiers d'entre elles environ sont rangées parmi le travail non-métropolitain. La proportion est renversée concernant les PCS les moins favorisées dont une part équivalente accède cependant au travail métropolitain.

(*) c'est à dire les secteurs suivants de la NAP99: 5, 6, 7, 17 à 19, 23 à 29, 31, 33, 34, 43, 51, 57 à 60,68 à $81,83,87$ à 90 . 
En observant de manière plus précise les données, on mesure aussi le poids de la crise de restructuration industrielle qui pèse sur les données du début des années 80. En effet, alors que l'ensemble du trafic lié aux activités du secteur «périproductif » (métropolitain ou non) et à la «reproduction sociale » croissent respectivement de $61 \%$ et de $48 \%$, l'évolution du trafic lié aux activités productives est de $+5 \%$ seulement entre 1980 et 1985, tant au sein du travail métropolitain qu'au sein du travail non-métropolitain. Une décennie après, sur la façade atlantique, la hausse de trafic due au travail productif métropolitain sera de +22\% de 1989 à 1993 alors que le travail productif non-métropolitain sera en baisse de près de $20 \%$. Cette dissociation des deux composantes des activités productives au cours des années 80 traduit l'approfondissement de la globalisation dont la spécialisation de l'Europe occidentale dans les productions à haute valeur ajoutée est un aspect important. En revanche, la crise de restructuration industrielle des années 70 et du début de la décennie 80 , si elle a constitué une étape préparatoire de cette spécialisation, a affecté l'outil industriel dans son ensemble.

Ainsi, on peut avancer que les résultats obtenus sur le TGV sud-est n'infirment pas ceux de l'Atlantique. Ils les « situent » d'un point de vue historique, permettant peut-être encore mieux de rattacher le dynamisme spécifique du trafic « métropolitain » aux tendances contemporaines de l'économie globale. L'analyse comparée des résultats des enquêtes TGV paraît renforcer le schéma d'une mise en place progressive, au cours des années 80, d'une économie métropolitaine fondée sur la manipulation de l'information. À travers cette dynamique, on peut alors mieux comprendre le sens des fluctuations de trafic constatées. On vérifie ainsi que le contexte macro-économique de ces observations de la mobilité à grande vitesse joue un rôle déterminant dans leur interprétation (KLEIN, 1998). Enfin, on constate surtout que se met en place, au fil des années 80 et 90 , une nouvelle modalité de différenciation de la mobilité. On va maintenant chercher à vérifier si cette différentiation sociale peut aussi se décliner au niveau spatial.

\section{Demande de déplacements à grande vitesse, espace métropolitain et espace banal}

Pour Félix DAMETTE et ses collègues, la notion de travail métropolitain a par essence une dimension spatiale affirmée puisqu'elle est définie sur la base du référent spatial parisien. C'est sur ce fondement que, dans son Atlas La France en villes, il choisit de qualifier de métropoles sept agglomérations de Province. Aussi est-il intéressant d'observer si le poids du travail métropolitain dans les flux de voyageurs circulant à grande vitesse est spatialement différencié et si ces différences sont cohérentes avec la structure urbaine analysée par Félix DAMETTE.

Pour ce faire, on s'appuiera sur l'enquête-TGV réalisée sur la zone Atlantique car elle couvre, entre Paris et Province, un ensemble de relations touchant des villes de tailles et de caractéristiques variées. On ne retiendra que les seuls résultats de la vague de 1993, obtenus après la mise en service du TGV-A. La principale motivation de ce choix, outre la simplification des résultats, est la volonté de concentrer l'analyse sur la demande qui s'exprime sur les moyens de déplacement à grande vitesse. II est clair que la situation de l'offre ferroviaire en 1989, donc avant la mise en service de la ligne nouvelle, ne permettait pas de répondre à ce souhait.

\section{Trafic métropolitain entre métropoles ?}

Dans une première étape, on observe donc le pourcentage représenté par les « travailleurs métropolitains » dans l'ensemble du trafic d'affaires observé pour 
plusieurs relations joignant la façade ouest de la France à Paris. Les liaisons représentants les distances les plus longues, concernant les trois départements de la péninsule bretonne d'une part et ceux situés au sud-ouest au-delà de Bordeaux d'autre part ne figurent pas dans ces résultats. II s'agit d'abord de ne conserver que des trajets effectués pour l'essentiel en moins de trois heures, et ensuite de sauvegarder des zones relativement homogènes (la diminution des flux avec la distance et la nécessité de ne travailler qu'avec des sous-ensembles conservant un effectif raisonnable impliqueraient en effet la constitution de zones très disparates pour représenter les départements les plus éloignés de Paris). Toulouse, qui est un gros émetteur de trafic et dont les relations d'affaires sont assurées à 90\% par avion, peut en revanche figurer. Le tableau 3 distingue d'un côté les plus grandes agglomérations auxquelles Félix DAMETTE a attribué un caractère métropolitain, et de l'autre les espaces a priori plus ordinaires. II faut encore préciser que les informations spatiales ont été recueillies à l'échelle départementale. Chaque département est ensuite assimilé à sa préfecture.

Tableau 3 : Part du travail métropolitain dans le trafic d'affaires tout mode selon la liaison avec Paris dans l'enquête TGV-A de 1993

\begin{tabular}{rr}
\hline Bordeaux & $74 \%$ \\
Nantes & $64 \%$ \\
Rennes & $80 \%$ \\
Toulouse & $73 \%$ \\
\hline
\end{tabular}

\begin{tabular}{rr}
\hline Le Mans & $56 \%$ \\
Tours & $67 \%$ \\
Poitiers & $63 \%$ \\
Angers et Laval & $54 \%$ \\
Niort et La Roche-s/Yon & $75 \%$ \\
Angoulême et La & $55 \%$ \\
Rochelle & \\
\hline
\end{tabular}

Sur toutes les relations, les activités métropolitaines génèrent plus de la moitié, et même souvent plus de $60 \%$, du trafic d'affaires. Cette part élevée, qui avait été mesurée sur les trafics globaux dans la section précédente, se trouve ici confirmée sans souffrir d'exception spatiale. Plus précisément, il apparaît que les trafics concernant les agglomérations les plus importantes montrent plutôt des taux de travail métropolitain plus importants que ceux touchant les zones dépourvues de très grandes villes.

Deux chiffres semblent contredire cette observation : le taux plutôt faible obtenu par Nantes, celui, très élevé, affiché par Niort et La Roche-s/Yon. II semble qu'ils puissent s'expliquer par des particularités locales sans pour autant remettre en cause la conclusion générale. Les données disponibles permettent en effet de décomposer les chiffres présentés de manière à ventiler travail métropolitain et travail non- métropolitain par secteur d'activité. Cette décomposition est rapidement délicate en raison d'effectifs qui deviennent insuffisants. Néanmoins, elle permet de mieux comprendre les deux résultats particuliers qui font exception ici.

Le score de Nantes par exemple, s'explique ainsi par la faible représentation (par rapport aux trois autres métropoles observées) des branches industrielles techniciennes selon la terminologie du laboratoire STRATES, c'est-à-dire essentiellement les industries de pointe. Ces branches, qui contribuent à hauteur d'environ $20 \%$ au travail métropolitain à Bordeaux, Toulouse ou Rennes, pèsent près de deux fois moins à Nantes. En revanche, les branches du travail non-métropolitain des industries qualifiées ou spécialisées, qui regroupent les industries traditionnelles, 
ainsi que la branche agro-alimentaire, apparaissent plus présentes dans les trafics nantais.

Cette image de Nantes comme ville d'industrie traditionnelle n'est pas totalement corroborée par les données que présente l'analyse exhaustive de Félix DAMETTE (1994). Pourtant, elle correspond à une part de réalité : Toulouse, mais aussi Bordeaux sont davantage connue pour leurs activités aéronautiques que l'estuaire de la Loire qui bénéficie dans ce secteur d'implantations moins importantes et moins diversifiées $^{2}$; Rennes peut se prévaloir de la présence d'une activité orientée vers les télécoms et l'informatique repérable dans les statistiques. Le marketing de l'agglomération ne se prive pas de le souligner. Toujours selon les données de I'INSEE en revanche, les industries dominantes en Loire-Atlantique sont, outre la construction navale, l'agro-alimentaire et la pétrochimie ${ }^{3}$.

Cela dit, cette parenthèse met surtout en exergue le caractère forcément arbitraire et discutable de tout classement sur base statistique, qui ici, aboutit à chercher du travail métropolitain dans certaines branches et pas dans d'autres. Elle appelle en fait un affinement de la définition du travail métropolitain. Deux faits essentiels sont à souligner concernant le trafic nantais. Le plus important tient à la vigueur des flux générés par les activités d'intermédiation. Le second est la diversité du tissu économique. Ce constat rejoint cette fois celui de Félix DAMETTE (1994, p. 48) et montre que, malgré ses spécificités industrielles, l'agglomération nantaise ne déroge pas à son rang de métropole de Province.

L'autre chiffre « gênant » est encore plus simple à interpréter. Le taux important de travail métropolitain de l'ensemble Niort + La-Roche-s/Yon est entièrement dû à l'hyper-spécialisation de la préfecture des Deux-Sèvres. Observée à travers les trafics de et vers Paris, l'activité de Niort possède en effet toutes les apparences d'une mono-activité dans le secteur des assurances. La circulation des cadres des sociétés d'assurance génère à elle seule près de $80 \%$ des flux. Elle résulte de la nécessité de gérer et de coordonner des fonctions intensives en emplois qui ont été déconcentrées dans une zone où les salaires sont moins élevés qu'en Île-de-France. Au total, il semble bien que l'on puisse considérer les résultats constatés sur Nantes et Niort comme des cas particuliers qui ne remettent pas en cause le schéma général d'une différenciation spatiale légère mais réelle (de l'ordre de 10\%) de la répartition du travail métropolitain dans les trafics. Cette image obtenue à partir des flux de voyageurs apparaît alors cohérente avec celle reflétant la concentration spécifique du travail métropolitain dans les grandes agglomérations.

\section{Des trafics révélateurs des hiérarchies de l'espace productif}

Pour aller plus loin dans la compréhension de ces mouvements de personnes, on peut croiser ces résultats avec quelques caractéristiques des voyageurs. On cherchera alors à vérifier la mesure dans laquelle ces flux révèlent une hiérarchisation de l'espace. Dans cet esprit, on retiendra deux variables dont les

\footnotetext{
${ }^{2}$ Les tableaux économiques régionaux de L'INSEE indiquent des volumes d'emplois comparables dans le secteur "construction navale, aéronautique, armement" (poste 17 de la NAP 40) : 11800 emplois en 1993 en Loire-Atlantique, 8300 en Gironde et 12900 en Haute-Garonne (INSEE Pays-de-la-Loire, 1993 ; INSEE Aquitaine, 1994, INSEE Midi-Pyrénées, 1993). Mais l'activité de construction navale, qui ne donne pas lieu à travail métropolitain, pèse d'un poids important dans le seul le département de la Loire-Atlantique

${ }^{3}$ Le premier secteur d'activité industrielle de l'agglomération nantaise stricto sensu est, en termes d'emploi, celui de la construction électrique et électronique qui pèse pour $19 \%$ dans l'emploi industriel total (CETE de l'Ouest, 1997)
} 
diverses analyses menées sur ces résultats d'enquête ont déjà montré l'importance (KLEIN et CLAISSE, 1997). La première est naturellement la PCS. La seconde est le lieu d'habitation du voyageur en distinguant simplement les Parisiens qui se rendent en Province des provinciaux qui montent à Paris.

Compte tenu de la taille de l'échantillon observé qui ne permet pas de conserver des effectifs suffisants pour garantir la fiabilité des résultats dès que l'on distingue trop de sous-catégories, on utilise ici un jeu de quatre variables simplifiées, construites selon deux modalités chacune :

- La première porte sur la PCS du voyageur et distingue les PCS supérieures $(P C S+)$ des PCS inférieures (PCS-), ainsi que précisé dans la première section ;

- La seconde distingue le travail métropolitain (WMET) du travail non-métropolitain, que l'on peut proposer de dénommer aussi travail banal (WBAN) ;

- La troisième classe les voyageurs en fonction de leur lieu d'habitation, mais de fait, elle renseigne aussi sur le sens du voyage : la visite d'un provincial à Paris (PROV) ou la visite d'un Parisien en Province (PARI) ;

- La dernière porte sur le type d'espace qui génère ou reçoit l'échange avec Paris ; elle regroupe les 4 agglomérations les plus importantes de la zone d'un côté (Rennes, Nantes, Bordeaux et Toulouse) (AGGL) et l'ensemble des villes de moindre importance de l'autre (VILL).

Dans cet ensemble, la variable de PCS joue un rôle tout à fait particulier au regard de la question posée. En effet, I'hypothèse sous-jacente est que plus un flux de voyages de $A$ vers $B$ révèle un taux de voyageurs de PCS élevée, plus ce flux est porteur d'informations, de capacités de commandement ou de compétences de haut niveau. Sur cette base, on peut alors considérer deux situations-type.

La comparaison de la composition des flux de $A$ vers $B$ et de $B$ vers $A$ permet tout d'abord de révéler des liens de domination entre $A$ et $B$. Si, par exemple, le flux de $B$ vers $A$ est composé de voyageurs de PCS inférieure plus nombreux que celui de $A$ vers $B$, on peut induire que les échanges de personnes entre $A$ et $B$ traduisent une relation dissymétrique de domination des activités de l'espace $A$ sur les activités de l'espace B.

La comparaison de la composition des flux de voyageurs sur deux liaisons différentes ne révèle pas de lien de domination entre espace, mais plutôt une hiérarchie entre branches ou nœuds d'un réseau. Une proportion moins importantes de voyageurs de PCS élevée entre $A$ et $C$ qu'entre $A$ et $B$ indique en effet que les activités impliquant des déplacements entre $A$ et $C$ sont moins riches en informations sophistiquées et ou en capacité de commandement que celles générant les échanges entre $A$ et $B$.

Sur cette base, la population de voyageurs d'affaires observés en 1993 sur le TGV-A et les modes concurrents déjà utilisée pour le tableau 3 a fait l'objet d'une segmentation automatique en fonction de la variable PCS+/PCS- selon les trois variables explicatives WMETIWBAN, PARIIPROV et VILLIAGGL. Les étapes de cette segmentation et la répartition PCS+/PCS- à chacune d'elles sont présentés dans le schéma 1. Pour la dernière étape, le rapport des volumes de flux PARIIPROV est également indiqué. 


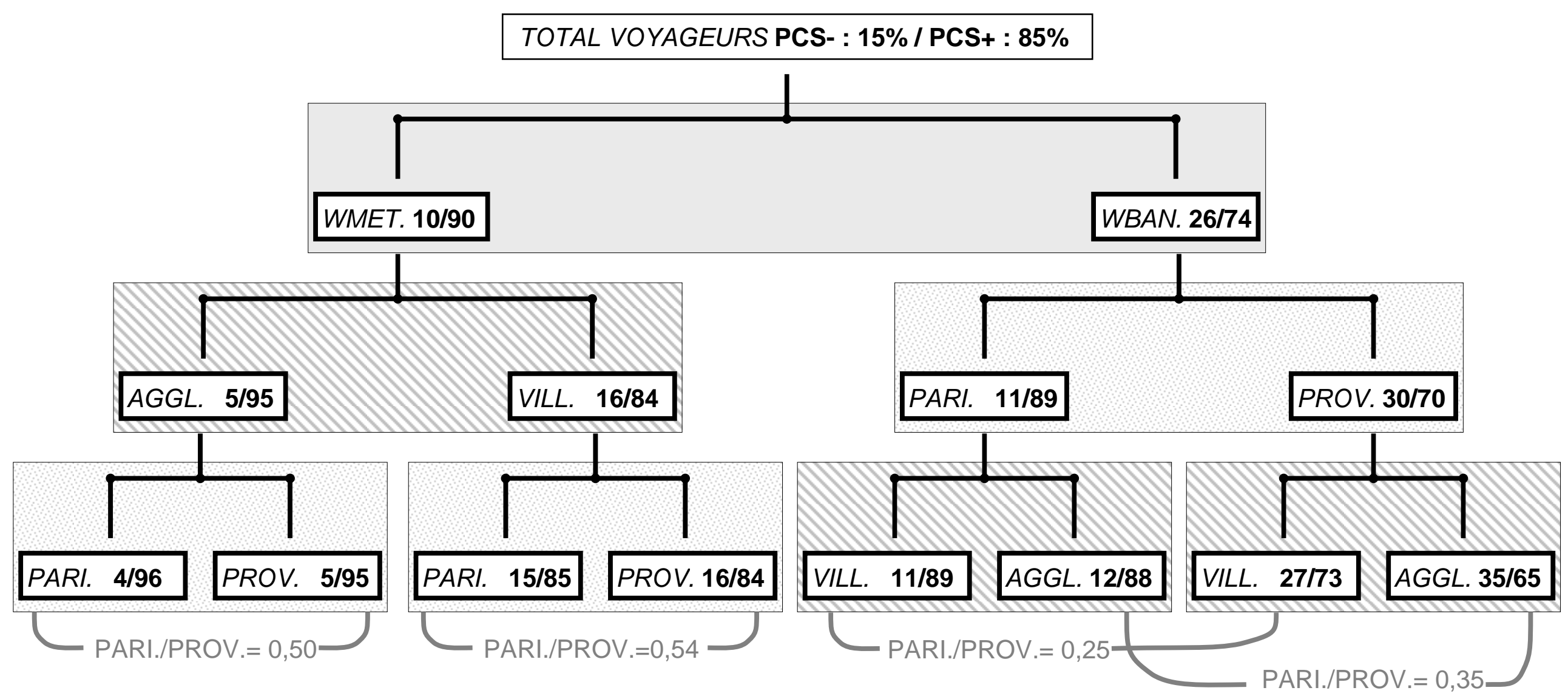

Shéma 1 : Segmentation d'une population de voyageurs d'affaires entre Paris et l'ouest de la France selon la PCS.

Sources : enquête TGV-A 1993, Laboratoire d'Economie des Transports 
Partant d'une population initiale ressortissant à $85 \%$ des $P C S+$, la première étape de la segmentation distingue sans surprise le travail banal (WBAN), comptant un quart de PCS-, du travail métropolitain (WMET), n'en comptant que 10\%. Concernant le travail banal, la seconde étape différencie le sens du déplacement: les Parisiens descendant en Province (PARl) appartiennent à $90 \%$ aux catégories socioprofessionnelles élevées alors que les provinciaux (PROV) de niveau équivalent montant à Paris ne font que $70 \%$ du total. Au sein du travail banal, les flux sont donc très déséquilibrés suivant le sens de déplacement. La troisième étape de segmentation, indiquée ici pour illustration, montre que la distinction VILL/AGGL n'a dans ce cas aucune influence sur la composition sociale des flux de voyageurs.

Compte tenu des hypothèses posées ci-dessus, on peut interpréter la nette prépondérance des PCS+ chez les Parisiens comme le signe d'une domination des espaces non-métropolitains de Province par les structures de coordination parisiennes. Cette domination est accompagnée d'un très fort déséquilibre en volume, toujours suivant le sens de déplacement, puisque pour trois provinciaux ressortissant du travail banal montant à Paris au départ d'une grande agglomération, seulement un Parisiens effectue le trajet dans l'autre sens et que cette proportion descend même à quatre pour un si l'on considère les autre villes de Province.

Ce schéma de domination de l'espace banal par un espace métropolitain est largement cohérent avec celui proposé par Saskia SASSEN. La lecture parisienne de l'espace non-métropolitain n'est cependant pas à prendre au pied de la lettre : rien n'indique que cette domination ne transite pas également par d'autres relais, en Province, situés notamment dans les plus grandes agglomérations. Les données utilisées ici ne permettent pas de le vérifier, ni de l'infirmer.

Concernant le travail métropolitain, l'autre partie du schéma 1 donne à voir une structure identique de contraste entre deux configurations de la répartition sociale des voyageurs. Mais dans ce cas, la seconde étape de segmentation distingue AGGL (avec un taux de PCS+ de 95\%) et VILL (avec seulement $84 \%$ de PCS+). En troisième étape, le sens de déplacement n'a plus aucune influence sur le niveau hiérarchique des flux. En outre, les flux apparaissent quantitativement moins déséquilibrés : les provinciaux qui montent à Paris ne sont «que » 1,8 à 2 fois plus que les Parisiens qui descendent en Province.

Au sein du travail métropolitain, cette situation où les flux de Parisiens et de provinciaux ne présentent pas de particularité du point de vue de leur composition n'est pas interprétable en termes de domination de Paris sur la Province. Le clivage s'opère cette fois autour du type d'espace de Province dont on observe les échanges avec Paris. Le phénomène de différenciation spatiale révélé ici distingue les grandes agglomérations de Province des plus petites villes. L'image produite reprend celle d'un réseau métropolitain hiérarchisé, mais les modalités selon lesquelles s'organisent les différences sont très différentes de celles qui prévalaient pour les activités banales.

C'est l'insertion dans des systèmes d'échange d'un niveau supérieur qui paraît distinguer les grandes agglomérations et Paris d'une part, des plus petites villes d'autre part. S'il fallait continuer intuitivement la figure qui se dessine, on pourrait sans aucun doute relier Paris à quelques autres capitales mondiales par des flux liés au travail métropolitain d'un niveau qui n'est pas atteint entre Paris et les grandes agglomérations françaises. De même, les échanges, que l'on n'observe pas ici, entre grandes agglomérations et plus petites villes devraient se situer à un niveau proche de ceux repérés entre Paris et ces plus petites villes. 
En revanche, à chacun des niveaux, les flux ne semblent pas trahir de dissymétrie fonctionnelle, de relation de commandement dans un sens et de subordination dans l'autre. Bien sûr, il convient de prendre cette dernière assertion avec prudence car il faut bien envisager que des échanges se produisent entre les différents niveaux du réseau métropolitain et que ces échanges soient orientés. Les données utilisées ici ne permettent guère que des hypothèses.

\section{Conclusion}

Cet article a été présenté comme une tentative d'importation de concept depuis la géographie du système productif vers l'analyse de la mobilité. De tels échanges entre champs d'analyse distincts ne sont pas dénués d'intérêts réciproques. Ici, la notion de travail métropolitain a d'abord été soumise à un test exigeant du point de vue de son opérationalité. En effet, les enquêtes-TGV n'ont absolument pas été conçues dans l'optique de son usage. D'ailleurs, les questionnaires ont été rédigés avant que les travaux du Laboratoire STRATES ne soient connus, ni même entamés en ce qui concerne l'enquête sud-est. C'est donc à partir d'un matériau très ordinaire, sans adaptation ex ante particulière, que cette nouvelle segmentation a pu être mise en œuvre. En outre, les données relatives au TGV-A se sont révélées, au traitement, extrêmement délicates en raison notamment de la diversité des situations observées. La variabilité, en particulier spatiale, de plusieurs résultats a priori importants a limité à l'époque la finesse possible des traitements de données ${ }^{4}$. Le fait que la notion de travail métropolitain demeure non seulement lisible, mais tout à fait pertinente dans le cadre d'une désagrégation spatiale n'a pas été une petite surprise. II s'agit d'un gage de robustesse qui permet d'envisager d'autres applications.

En second lieu, l'application «expérimentale » du concept théorisé de travail métropolitain apporte un certain nombre de confirmations. Jean GoTTMANN (1961), Félix DAMETTE (1994) ou Nadine CATTAN (et alii, 1994) par exemple, on déjà montré l'importance de l'observation des flux d'échanges entre les concentrations urbaines pour mettre en évidence la structure résiliaire de leur fonctionnement. Pour riches qu'elles soient, ces approches restent pour l'immense majorité cantonnées à l'analyse des volumes de déplacements en raison de la nature des données généralement disponibles. L'utilisation des données issues des enquêtes TGV permet d'avoir, à travers les caractéristiques des voyageurs, une image du contenu des déplacements réalisés. Même si cette source présente encore beaucoup de limites, même si elle n'a pas été complètement exploitée sous l'angle du travail métropolitain - puisque des renseignements relativement précis concernant le motif de déplacement sont également disponibles - c'est donc sur une information plus riche que peuvent être envisagés les phénomènes de métropolisation.

De fait, les flux de déplacements à grande vitesse du travail métropolitain et ceux des activités banales apparaissent nettement différenciés, notamment par leur dynamique et leur inscription dans l'espace productif. Ils permettent non seulement d'observer des structures spatiales bien particulières, mais offrent aussi une interprétation de ces phénomènes en termes de réseau au sein de l'espace métropolitain et de domination de l'espace métropolitain sur l'espace banal. À travers ces analyses, on retrouve en France une organisation spatiale du phénomène de globalisation, là où l'observation des simples volumes de déplacements conduit souvent à une représentation essentiellement gravitaire.

D'un autre point de vue, cet article répond à des préoccupations qui concernent la compréhension des flux de déplacement dans une optique plus ou moins immédiate

\footnotetext{
${ }^{4}$ Ces limites sont reprises dans le chapitre conclusif de (KLEIN et CLAISSE, 1997)
} 
d'évaluation des grands investissements. De ce point de vue, la lecture parallèle des résultats traditionnellement obtenus à partir de la PCS des voyageurs et de ceux issus de la segmentation entre travail métropolitain et travail banal est riche d'enseignements.

La question n'est pas de remettre en cause le constat d'une relative « démocratisation » de la vitesse à la faveur de la mise en service du TGV sud-est, ni celui du caractère nettement ségrégatif de l'usage du TGV-A en 1993. Les deux tendances - diffusion et hiérarchisation - sont inscrites sur le long terme dans l'histoire de la vitesse. II s'agit plutôt de tenter de percevoir la construction sociale à laquelle participe la mobilité ferroviaire à grande vitesse. C'est par rapport à cet objectif que la notion de travail métropolitain trouve un intérêt.

En passant les données au filtre d'une problématique économique et géographique, elle met au premier plan de l'analyse les structure sociales et spatiales - enfin explicitées dans ce cadre - des populations, des activités et des territoires que dessert le TGV. Sur la base de cette explicitation des déterminants sociaux - qu'il convient de compléter par l'explicitation tout aussi rigoureuse des déterminants de l'offre de transport - on peut alors envisager de construire une représentation des interactions qui réunissent transport, espace et société. Cette démarche participe ainsi au dépassement des lectures techno-déterministes auxquelles donne souvent prise le champ des transports.

\section{Bibliographie :}

R. A. BeAuregard (1990), "Trajectories of neighborhood change: the case of gentrification" Environment and Planning A, vol. 22, pp. 855-874.

Pierre Beckouche et Félix Damette (1993), "Une grille d'analyse de l'emploi : Le partage géographique du travail", Economie et statistique, $\mathrm{n}^{\circ} 270$, pp. 37-50.

Alain BonNAFOus (1987), "The regional impact of the TGV", Transportation, Vol.14, $\mathrm{n}^{\circ} 2$, pp.127-137.

Michel BraIBANT (1982), "Le tertiaire insaisissable ?", Economie et statistique, $\mathrm{n}^{\circ}$ 146, pp. 3-18.

Manuel CASTELLS (1996), The rise of the network society, Blackwell, Oxford. Traduction française par Philippe Delamare, 1998, L'ère de l'information, tome 1, La société en Réseaux, Fayard, Paris, 613 p.

Nadine Cattan Denise Pumain, Céline Rozenblatt et Thérèse SaINT-Julien (1994), Le système des villes européennes, Anthropos/Economica, coll. Villes, Paris, 201 p.

CETE de l'Ouest (1997), Nantes, 7 ans après l'arrivée du TGV-Atlantique, Ministère de l'Equipement/Direction des Affaires Economiques Internationales, 174 p.

Daniel COHEN (1994), Les infortunes de la propérité, Julliard, Paris. Edition de poche, 1997, Pocket, coll. Agora, Paris, 230 p.

Daniel CoHEN (1997), Richesse du monde, pauvretés des nations, Flammarion, coll. Champs, Paris, $167 \mathrm{p}$.

Kenneth E. COREY (1982), "Transactional forces and the metropolis", Ekistics, vol. 49, $\mathrm{n}^{\circ} 297$, pp. 416-423. 
Félix DAMETTE (1994), La France en ville, La documentation française, DATAR, coll. Informations et analyses, Paris, $271 \mathrm{p}$.

Susan FANSTEIN et Michael HARLE (1996), "Reprise et récession dans les villes internationales : Londres et New-York à l'ère contemporaine", Espaces et Sociétés, n॰82-83, 1-2/1996, pp. 167-191.

Jean-Michel FOURNIAU (1988), La genèse des grandes vitesses à la SNCF, de l'innovation à la décision du TGV sud-est, rapport INRETS $n^{\circ} 60$, INRETS, Arcueil, $174 \mathrm{p}$.

Jean GotTMANn (1961), Megalopolis: the Urbanized Northeastern Seaboard of the United States, New-York, Twentieth Century found, XI + 810 p.

Jean GotTMANN (1970), "Urban centrality and the interweaving of the quaternary activities", Ekistics, vol. 29, n 174, pp. 322-330.

INSEE Pays-de-la-Loire (1993), Tableaux économique des Pays-de-la-Loire, 207 p.

INSEE Midi-Pyrénées (1993), TEMP-Tableaux économique Midi-Pyrénées, 155 p.

INSEE Aquitaine (1994), Tableaux économique de l'Aquitaine 1994, 200 p.

Philippe JULIEN (1996), "Spécificité des grandes villes de province", Economie et statistique, $\mathrm{n}^{\circ}$ 294-295, pp. 137-152.

Olivier KLEIN (1998), "Les modifications de l'offre de transport: des effets automatiques à l'innovation socio-technique - l'exemple de la grande vitesse", Espaces et Sociétés, n 95, pp.95-126.

Olivier KLEIN (2001), Les horizons de la grande vitesse : le TGV, une innovation lue à travers les mutations de son époque, Thèse de Doctorat es sciences économiques, Université Lyon 2, 308 p.

Olivier KLEIN, Gérard ClAISSE, avec la collaboration de Pascal POCHET (1997), Le TGV-Atlantique: entre récession et concurrence, Laboratoire d'Economie des Transports, coll. Etudes \& Recherches, $\mathrm{n}^{\circ} 7$, Lyon, $163 \mathrm{p}$.

Paul R. Krugman (1996, Pop Internationalism, M.I.T. Press, Cambridge (Mass.). Traduction française de Anne SAINT-GIRONS (1998), La mondialisation n'est pas coupable: vertus et limites du libre-échange, La Découverte, coll. Textes à l'appui/série économie, Paris, 218 p.

Laboratoire STRATES (1989), Métropolisation et aires métropolitaines: Internationalisation et enjeux urbains, Rapport pour la DATAR, le Commisariat Général au Plan et le Plan Urbain, Paris, 355 p.

L. M. MCDOWELL (1997), "The new service class: housing, consumption, and lifestyle among London bankers in the 1990s", Environment and Planning A, vol. 29, pp. 2061-2078.

Jacob Meunier (2002), On the Fast Track - French Railway Modernization and the Origin of the TGV, 1944-1983, Praeger, Westport (Conn.), 255 p.

Petros Petsimeris et Susan BALL (2000), "Greater London entre hétérogénéité ethnique et polarisation socio-spatiale", Cybergéo, $\mathrm{n}^{\circ} 122$. Document électronique consulté le 02 novembre 2000 à l'adresse suivante : http://www.cybergeo.presse.fr/culture/ball/london.htm 
François PLASSARD (1987), Les effets du TGV sur les déplacements d'affaires entre Paris et Rhône-Alpes, Communication à la cinquième conférence internationale sur les comportements de déplacements, Aix-en-Provence, 20-23 octobre, $10 \mathrm{p}$.

Robert REICH (1991), The Work of Nations, Alfred A. Knopf, New-York. Traduction française par Daniel TEMAM, 1997, L'économie mondialisée, Dunod, Paris, 336 p.

Saskia SASSEN (1991), The global city, Princeton University Press. Traduction française par Denis-Armand CANAL, 1996, La ville globale, Descartes \& Cie, Paris, $530 \mathrm{p}$.

Pierre VeLtz (1996), Mondialisation, Villes et Territoires: L'économie d'archipel, Presses Universitaires de France, coll. Economie en liberté, Paris, 262 p. 